\title{
LA GINECOBSTETRICIA EN BOGOTA DURANTE EL SIGLO XIX
}

\section{Dr. Fernando Sánchez-Torres*}

Pasada la gesta emancipadora y organizada la República, en la administración del General Santander se creó la Universidad Central y como sección de ella una Escuela de Medicina, la cual empezó a funcionar desde 1827.

El Artículo 216 del Decreto del 3 de Octubre de 1826 sobre Plan de Estudios, contemplaba no solo la creación formal de la cátedra de Obstetricia sino que encargaba a la Facultad de promover la buena marcha de las Escuelas de Medicina (preludio de la actual Asociación Colombiana de Facultades de Medicina), examinar sangradores, parteras, farmacéuticos, y expedirles diplomas.

En 1833, y a instancias de la Facultad de Medicina, el gobierno emprendió activas medidas encaminadas a prohibir el ejercicio médico a toda persona que no tuviese título. En ese año, y por primera vez en Colombia, fueron habilitadas por el Estado algunas mujeres para desempeñar la profesión de parteras. Fueron ellas Juana Solórzano, Manuela Rodríguez e Isabel Cortés, a quienes apenas les era permitido recibir la criatura, ligar y cortar el cordón umbilical.

"Estas saludables disposiciones — comentaba Ibáñez algunos años después- sobre el difícil arte de los partos fueron descuidadas posteriormente y muchas mujeres del pueblo, careciendo de instrucción científica, se cledicaron a él, causando graves males a las confiadas parturientas que se ponían en sus manos". En efecto, desde 1850, por ley del 14 de Mayo, se declaró libre la enseñanza de la medicina y demás ramas afines, ley que acabó con la Escuela Universitaria; se auspició así nuevamente el libertinaje para recetar, abrir farmacias, ejercer la obstetricia, etc.

El 18 de Abril de 1852 hizo su aparición la primera publicación médica colombiana: La Lanceta, fundada y redactada por los doctores Antonio Vargas Reyes y Antonio Vargas Vega. Aquel día vio la luz pública también el primer artículo sobre temas ginecológicos, titulado "Enfermedades de la mujer". El operador del caso descrito en dicho artículo fue el doctor Vargas Reyes, quien extirpó un pólipo uterino a "una señora muy conocida en esta ciudad". Siendo este un hecho importante, transcribimos un aparte del mencionado escrito: "Puesto el espéculum de dos ramas, pudo verse el fondo del pólipo en el que se implantaron unas fuertes pinzas de Mousseaux, i a la vez que se efectuaban tracciones suaves con el fin de atraer el tu-

* Profesor Asociado del Departamento de Obstetricia y Ginecología. Facultad de Medicina de la Universidad Nacional de Colombia. 
mor al esterior, el hábil cirujano que mantenía el espéculum, lo retiraba gradualmente. Cuando ya se obtuvo, nuevas pinzas lo agarraron por los lados i en mui pocos instantes la totalidad del pólipo, junto con el cuello de la matriz i su fondo medio invertido, estuvieron fuera de los órganos sexuales. Unas grandes tijeras curbas sobre el plano lo desprendieron, i el útero recuperó su posición en el momento.

Según un testigo presencial "la operación fue practicada en menos de seis minutos, la paciente poco sufrió y la pérdida de sangre no pasó de cuatro onzas".

Aunque, como hemos visto, desde 1827 se creó la cátedra de obstetricia, no existía un lugar destinado para su enseñanza práctica pues el Hospital San Juan de Dios, única institución asistencial de entonces, de todo tenía menos de hospital. Esto no es una exageración; oigamos a Cordovez Moure: "Propiamente hablando, el establecimiento tenía más analogía con el Caravanserail de los árabes que con los hospitales de beneficencia. La mayor parte de las piezas altas estaban ocupadas por gentes menesterosas de ambos sexos, que entraban y salían cuando les daba la gana; en los pocos salones destinados para enfermería había, a lo largo de las paredes, cujas de madera barnizadas de rojo, con alto respaldo, en el cual se veía el número que correspondía al desgraciado enfermo que allí caía. Debajo de cada cama guardaban estos los trastos que necesitaban, junto con los víveres, el carbón y otros objetos que les servían para preparar los alimentos o aguas cocidas, todo lo cual daba a las enfermerías el aspecto de cocinas por el humo del combustible". Hasta aquí la descripción de Cordovez. Y pensar que allí, en tan desapacible ambiente, el doctor Leoncio Barreto el 3 de Octubre de 1864 llevó a cabo, con todo éxito, la primera ovariectomía practicada en Colombia.

En 1865 la Comisión Inspectora del Hospital propuso que este se unie:ra a la Escuela de Medicina privada que fundara un año antes el doctor Antonio Vargas Reyes, cediendo un anfiteatro y los cadáveres de que se pudiera disponer, un salón para grados y exámenes y las salas para las clases teóricas, a cambio de que se le dieran, anualmente y sin remuneración alguna, dos médicos, dos cirujanos, un farmacéutico, dos practicantes y un enfermero mayor. Además, independientemente de las variaciones que adoptara la Escuela en sus programas, se exigía la creación de una cátedra de Clínica y otra de Obstetricia. Pero todavía para 1867 - año en que se expidió la ley que creó la Universidad Nacional_- el Hospital no contaba con un servicio de Maternidad, pues en el decreto del 3 de Enero de 1868, que reglamentó dicha ley, se anota que el profesor de Obstetricia haría la clase de Clínica Obstétrica "si se llegaba a establecer en el hospital una sala de maternidad".

Tan notorio sería el abandono en que se hallaba el ejercicio obstétrico que el 20 de Marzo de 1869 José Peregrino Sanmiguel, connotado homeópata de la ciudad, se dirigió al Congreso para solicitar la creación de un "Colegio nacional de profesoras para el servicio de las enfermedades de la mujer y de los niños". En su exposición de motivos decía: "Las mujeros $\mathrm{i}$ niños que perecen en los pueblos de la República abandonados a la naturaleza; a las prescripciones de parteras ignorantes i de curanderos sin ciencia, exigen del Gobierno una 
pronta medida. No solamente parteras medianamente instruídas, sino profesoras ilustradas son las que se deben mandar a los Estados, para que en escuelas costeadas por los Gobiernos locales se eduquen las destinadas a todas las parroquias, en las que legarán su ciencia a discípulas idóneas, i estas a otras sucesivamente... La ciencia que adquieran las alumnas del Colejio Nacional homeopático, vale más que la riqueza inagotable de nuestras minas..."

Planta exótica en aquellas calendas era el médico especialista. "Nuestros médicos - decía Pedro María lbáñez - se ven obligados a recetar diariamente ya un caso de patología interna, ya a practicar una operación, - a prestar los auxilios de la ciencia en un caso de distocia". Confirma esta aseveración la anécdota relatada por el autor de las Reminiscencias de Santafé y Bogotá, de la cual fue personaje central el cirujano escocés $\mathrm{Ni}$ nian Ricardo Cheyne, quien llegó al país en 1824 y falleció en la capital en 1872. "Entre las muchas obras de caridad levadas a cabo por el doctor Cheyne -escribe Cordovez- citaremos el caso de una mendiga que no podía dar a luz, e imploraba auxilio en un rancho levantado con desperdicios de la plaza de mercado en las orillas del río San Francisco, entre los puentes Nuevo y el Telégrafo. Al pasar cerca de allí el doctor, oyó voces lastimeras y acudió al lugar de donde éstas salían. Impuesto el generoso médico de lo que se trataba, despachó al sirviente con el caballo y la orden de que le trajera los instrumentos adecuados a la operación indispensable para salvar la vida de aquella infeliz. Allí, en esa miserable vivienda, permaneció el sabio profesor hasta que sacó del tarnce a la madre y al hijo; compró personalmente, en una chichería inmediata. una taza de caldo que hizo tomar a la paciente, le dejó algunos reales de limosna, pagó a una mujer vecina con el fin de que cuidara a la enferma, se lavó las manos en el río y volvió a su casa al amanecer del día siguiente. La favorecida no fue ingrata y pregonó al beneficio recibido".

No faltaron, sin embargo, médicos que mostraron inclinación por las disciplinas ginecobstétricas. Por los documentos que hemos podido consultar fue el doctor Joaquín Maldonado, natural de Chocontá, uno de los primeros especialistas de la tocología. Y lo creemos porque sus colegas lo tenían como persona que poseía del ramo obstétrico extensos y sólidos conocimientos. $Y$ había de ser así pues fue profesor de Obsterticia en el Colegio Mayor del Rosario desde 1849. También ocupó las cátedras de Terapéutica y Anatomía. Retirado voluntariamente de la actividad docente en 1856, se consagró al estudio de las ginecopatías y a la atención de partos. Parece, pues, que el doctor Maldonado fue el primer especialista "integrado" en Obstetricia y Ginecología.

En Bogotá residía, hasta el año de 1866 en que murió, el doctor José Salvador Riera, natural y médico de La Habana, quien figuraba como especialista en enfermedades de la mujer. Entre 1860 y 1863 fueron impresos dos trabajos suyos: "La esterilidad en ambos sexos" y "Las enfermedades de las mujeres". Desafortunadamente de estas publicaciones no quedó constancia distinta a la referencia que de ellas hace Pedro $M$. lbáñez.

Procedente de París, en donde había cursado estudios profesionales, se radicó en la capital, en 1870, la doctora Ana Galvis, colombiana de ori- 
gen. Durante los pocos años que ejerció en Bogotá se anunció como "especialista en enfermedades del útero y sus anexos". Creemos que la doctora Galvis, además de corresponderle el honor de ser la primera médica que ejerció en Colombia, fue también la primera especialista en Ginecología.

Librado Rivas, nacido en Bogotá en 1822 y graduado en la Universidad Central en 1846, ocupó diversas cátedras médicas desde 1855 hasta 1877, año de su muerte. Fue profesor de Maternidad, sobresaliendo por su habilidad para ejecutar toda clase de intervenciones obstétricas.

Natural de Chaparral, Rafael Rocha Castilla hizo estudios de medicina en Bogotá para refrendar su título en París. Dictó Obstetricia en el Colegio del Rosario durante cinco años (1860-1865); luego ocupó la cátedra de Anatomía durante 16 años. En 1881 viajó a Europa donde se radicó por algún tiempo.

Aunque ignoramos si en el transcurso de su ejercicio profesional practicaron con preferencia la ginecobstetricia, es necesario mencionar los nombres de algunos colegas graduados en París con tesis relacionadas con la especialidad. Rafael Grau, doctorado en 1854, escribió un trabajo sobre fístulas vésicouterinas; Joaquín Vengoechea se recibió en 1857 con un estudio sobre "Tratamiento de los quistes del ovario"; la tesis de Juan de Dios Uribe, presentada en 1874, versó acerca de "Las causas y los mecanismos de las hemorragias intersticiales de los órganos genitales de la mujer", y la de Nicanor Insignares, en 1875, sobre la "Relación de la endocarditis subaguda con la hemiplejía puerperal". Ignacio Gutiérrez Ponce, graduado en París, fue profesor de Medicina en Nueva York, en 1874, con un trabajo, muy elogiado por cierto, sobre "Fiebre puerperal".

Forzoso es hacer mención especial, aun cuando sea somera, de las vidas y hazañas de Leoncio Barreto, José María Buendía y Juan Evangelista Manrique, pues ellos, indudablemente, fueron los precursores de la Obstetricia y la Ginecología colombianas.

Leonci Barreto nació en Bogotá en 1839. Fue profesor de Obstetricia desde 1865 y de Clínica Obstétrica hasta 1901. Ocupó el cargo de Inspector de Estudios de la Facultad de Medicina. Como hemos comentado atrás, practicó por primera vez una ovariectomía, el 3 de Octubre de 1864, operación esta que se constituyó en la segunda laparotomía hecha en Colombia. En 1873, también de primero, ejecutó una sinfisiotomía, según la técnica de Sigault. A pesar de ser un cirujano y un obstetra de gran habilidad y experiencia, tuvo la debilidad de guardar para sí las proezas que hizo en el dilatado ejercicio de su profesión. Tal vez pecó por modesto. De no ser por Eliseo Montaña y por Luis Largacha, aquellas dos intervenciones, hitos en la evolución de la especialidad entre nosotros, hubieran pasado desapercibidas. Solo sabemos que escribió un trabajo titulado "Enquistamiento de la placenta antes del parto" y un "Compendio de Obstetricia para las comadronas.

En 1830 nació José María Buendía en La Flata, Municipio del entonces Estado Soberano del Tolima. Cursó Literatura en el Colegio Seminario de San Bartolomé de Bogotá y luego hizo estudios de Medicina en el Colegio Mayor del Rosario, recibiendo el doctorado en 1850. Durante cuatro años ejerció en diversas poblaciones 
de Antioquia y del Tolima, y en 1854 viajó a Europa para actualizar sus conocimientos. Un lustro más tarde regresó al país y se estableció en el Tolima; en 1864, en calidad de Representante al Congreso Nacional, volvió a Bogotá. Aquí tomó parte activa en la fundación de la escuela privada de Medicina y regentó en ella la cátedra de Obstetricia. Terminadas las sesiones del Congreso, viajó por segunda vez a Europa, de donde retornó en 1868 para radicarse definitivamente en la capital. Ocupó distintas cátedras, llegando a ser profesor titular por muchos años de la Clínica Obstétrica e Infantil. Fue Rector de la Facultad de Medicina de la Universidad Nacional desde 1892 hasta 1897 y presidió en dos ocasiones la Sociedad de Medicina y Ciencias Naturales. No dejó obra escrita alguna pero, en cambio, modeló a nuestros primeros obstetras, entre ellos a su propio hijo, el famoso Nicolás Buendía.

Juan Evangelista Manrique, nació en 1861 en la hacienda de La Herrera, municipio de Bojacá. Cursó estudios de Literatura y Filosofía en el Colegio de Luis Cuervo y se graduó de médico en la Facultad de Bogotá el 12 de Mayo de 1882. Viajó a París en donde refrendó su título con la tesis sobre tema ginecológico "Estudio sobre la operación de Alexander", que lo hizo acreedor al Lauro y Medalla de oro. Volvió a Bogotá en 1886 para ejercer en forma brillantísima por más de veinte años. Fue miembro fundador del Club Médico, de la Sociedad de Cirugía y del Hospital San José, institución ésta de la que fue su primer Presidente y redactor de sus estatutos y reglamentos. Se cuenta, con toda justicia, como uno de los iniciadores de la cirugía en Colombia y, por ende, de la ginecología. Bien sabemos que en aquellos años finiseculares la ginecología no era una especialidad sino que estaba involucrada dentro de la cirugía general. De ahí que podamos afirmar que fue el pionero de la cirugía ginecológica en Colombia.

Escuchemos por qué: El 6 de Junio de 1887 hace la primera histerectomía abdominal: el 2 de Abril de 1888, también de primero, ejecuta una miomectomía por vía vaginal; meses más tarde corrige un prolapso uterino con la operación de AlquiéAlexander, y en el mismo año lleva a cabo la primera histerectomía vaginal. Como si fuera poco dicta en su propia casa un ciclo de conferencias sobre temas ginecológicos, ya que la pasión política le había negado la cátedra universitaria. Solo hasta 1906 se le nombró profesor de Clínica Quirúrgica. El doctor Isaac Rodríguez, quien asistió a estas conferencias, recordaba así aquel hecho insólito: "...quería con anhelo que todos sus compañeros amantes del deber participaran de sus conocimientos, y entonces, cuando apenas se iniciaban los estudios de Ginecología en nuestra Facultad, sin átomo de egoismo, abrió en su casa un centro de conferencias magníficas en las que ostentaba su verbo poderoso, fácil $y$ ameno, y su erudición sobresaliente entre los estudiantes y colegas que nos complacíamos en oirlo...'

De su habilidad y criterio quirúrgicos da también cuenta el doctor Isaac Rodríguez en un discurso pronunciado en la Sociedad de Cirugía el 14 de Noviembre de 1914 y del cual transcribimos algunos apartes, tomados del libro de Laurentino Muñoz, "Historia del Hospital de San José": "Cuando apenas se iniciaba la época de la cirugía abdominal -finales del siglo- en El Campito de San José, en donde entusiasta oficiaba el doc- 
tor Manrique en beneficio de la ciencia y de los desheredados de la fortuna, se presentó una señora con un enorme tumor abdominal. Hecho el diagnóstico de fibroma uterino, se procedió a la histerectomía abdominal con todas las minuciosas exigencias de la cirugía moderna; abierta la cavidad peritoneal, que él, con su jovialidad característica, llamaba entonces "una caja de sorpresas", en compañía de su distinguido colaborador e ilustre fundador del "Repertorio de Medicina y Cirugía", doctor José María Montoya, procedió a la enucleación de aquel voluminoso fibroma rodeado de adherencias; a pesar de una cuidadosa disección, fue interesado uno de los uréteres cerca de su extremidad vesical (aquí la inesperada complicación). Inmediatamente el hábil cirujano diseca el tubo ureteral y lo sutura convenientemente a la extremidad inferior de la herida abdominal. Por varios días sigue el curso de la orina hacia el exterior sin peligro alguno para la operada y más bien tuvimos la ocasión de observar el hecho clínico de que la orina aséptica que humedecía los labios suturados no impidió en manera alguna su rápida cicatrización.

"Salvado el primer peligro, nuestro infatigable cirujano, ansioso de volver el uréter a su puesto y de impulsar el interés científico de sus compañeros por la clínica quirúrgica de la Sociedad de Cirugía, convocó galantemente a todos sus miembros a fin de estudiar y decidir la intervención más acertada. Entonces se optó por la operación conocida con el nombre de Ureterocistoneostomía, la cual fue practicada cuando la enferma se halló en las mejores condiciones para soportar una segunda laparotomía.

"Con la mayor pulcritud y habilidad se llevó a efecto esta delicada operación, insertando por medio de finas y adecuadas suturas la extremidad ureteral directamente al punto más conveniente de la vejiga, lo cual dio por resultado en pocos días la curación de su interesante operada y la dulce satisfacción del éxito del cirujano".

El doctor Juan E. Manrique murić en San Sebastián, España, el 13 de Octubre de 1914, en el desempeño de sus funciones diplomáticas.

Para terminar este capítulo diremos algo acerca de la manera como se atendía la obstetricia en Bogotá, claudicando ya el siglo XIX.

Bien se ve, por la tesis de grado de Enrique Sánchez, presentada en 1892 y titulada "Necesidad de reglamentar en Colombia el ejercicio de la profesión obstetrical", que no solo se insistía en la conveniencia de ejercer vigilancia sobre aquellas personas que de moiu proprio eran parteras, sino que se esbozaba ya la posibilidad de que se hiciera de la Obstetricia una especialidad de la medicina.

Por el hecho de haber crecido en número y calidad el cuerpo médico de la ciudad, las mujeres de superior condición cultural y económica procuraban que la atención de sus partos estuviera a cargo de los profesionales de la medicina. "Por fortuna - dice el tantas veces citado doctor Ibáñez - los consejos de los médicos han sido atendidos, a lo menos en la parte ilustrada de nuestra sociedad y actualmente ilustrados profesores, llenan en el lecho de dolor, las funciones delicadas que hasta ayer desempeñaron impropiamente las parteras".

Pero veamos cómo ejercían sus funciones estos ilustres colegas. Juzgamos que todo médico que se preciara de tocólogo, acudiría a la alcoba 
de su paciente llevando bajo el brazo el estuche o trousse obstétrico, igual o parecido a los que fabricaban Dubois y Galante e hijos en París. Todos los aparatos e instrumentos utilizados en obstetricia estaban contenidos allí: fórceps, embriótomo, cefalotribo, dilatadores, tijeras, sondas, estetoscopio, pelvímetro, etc. Era lógico, tenía que ir preparado para ejecutar, en un momento dado y sobre el mismo "lecho del dolor", las más difíciles intervenciones.

Además de su aparataje también había de llevar una buena dosis de paciencia pues era costumbre demandar su concurso prematuramente, cuando apenas se vislumbraba que el parto iba a iniciarse. "Al presentarse los primeros dolores -afirma el doctor Sánchez - sin detenerse a considerar si son sintomáticos de haber empezado el trabajo, o provenientes de otra causa, salen a buscarlo y lo hacen venir precipitadamente a colocarse al lado de la parturienta, en donde se le exige que permanezca día y noche por semanas enteras, a veces descuidando sus importantes quehaceres, hasta que termine el parto".

La alcoba destinada para el acto obstétrico era preparada de antemano. Aunque el vulgo acostumbraba cerrar las puertas y ventanas, cubriendo cuidadosamente las rendijas con sábanas y colchas, oigamos las recomendaciones que daba, para tal efecto, el doctor Carlos Ramírez: "El aposento que se destine al parto ha de ser una pieza espaciosa, amplia, seca, que pueda ventilarse bien pero sin que se esté expuesta a fuertes corrientes de aire, para lo cual debe estar separada del patio por una o dos piezas... Es de desearse que tenga un aspecto agradable, que sus paredes estén muy aseadas, y que, en caso de ser empapeladas, los colores sean suaves; el pavimento debe estar alfombrado o por lo menos cubierto con esteras para disminuir el ruido de los que caminan en ella".

Como se pensaba que la debilidad general podía ser causa de distocia de contracción, o "disodinia", prescribíase durante la gestación un régimen fortificante $y$ en el parto administrábanse estimulantes como la infusión de canela, té con brandy o vino seco, licor de Hoffman y caldos alimenticios, lo mismo que fricciones suaves y metódicas sobre el fondo del útero. Además, para reblandecer los tejidos y excitar las contracciones, se aconsejaba un baño de asiento, caliente. Para favorecer la evolución del trabajo de parto, los obstetras apelaban a recursos de orden psicológico. Así, por ejemplo, recomendaban que el médico serenara el espíritu de la parturienta, infundiéndole valor y confianza; debía distraerla hablándole de cosas agradables que alejaran de su mente las preocupaciones, que podían trocarse en causa de distocia. Era, indudablemente, una modalidad de lo que después iría a recibir el nombre de "transfusión de confianza", y más luego el de "acondicionamiento extemporáneo".

Desencadenado el trabajo y si había debilidad contráctil, se apelaba a la aplicación local de éter sulfúrico y al taponamiento de la vagina, que al decir de algunos, tenía magnífico efecto, lo mismo que la "cateterización del útero", método que consistía en introducir una sonda elástica dentro de la cavidad uterina. Probablemente la ruptura de membranas que había de producirse con este procedimiento, era realmente lo que mejoraba la dinámica. Durante el período expulsivo la maniobra de Kristeller era también socorrida. 
Como fármacos ocitócicos se empleaban, en primer término, los derivados del cornezuelo de centeno. Entre estos eran muy conocidos la ergotina de Ivon, que era un extracto acuoso y la ergotina de Wiggers, que era un extracto alcohólico. Sin embargo, a fines del siglo ya había disparidad de conceptos sobre la conveniencia o no de suministrarlos durante la gravidez. Por eso se preconizaban otras sustancias tales como el cannabis índica, el extracto de pulsatilla, la decocción de uva ursi, pero sus resultados eran muy inciertos. Más confianza se tenía en la ipecacuana y en las sales de quinina. La primera se usaba en dosis de 5 centígramos, repetida varias veces y a intervalos cortos. La quinina se administraba bien en cucharadas o en inyecciones hipodérmicas según las siguientes fórmulas que aconsejaba Froilán Mahecha en 1896:

Clorhidrato de quinina

Acido clorhídrico

Jarabe de menta

Agua destilada tos.

M. y R. "Cucharadas" .Una cada 10 minu-

\section{Esta otra:}

Sulfato de quinina

Agua destilada

Agua de Rabel

M. Y R. "Inyección hipodérmica".

En aquellos casos de "espasmos" se utilizaba la belladona en la siguiente forma:

Hojas de belladona

Agua caliente

H. inf. cuel. y agre. :

Vino de opio

1 gramo

10 gramos

1 gramo

R. "Inyecciones No 2". Para aplicarlas con diez minutos de intervalo.

También se usaban las enemas así:

Raíz de valeriana

Agua caliente
H. inf. cuel. y agre. :

Bromuro de potasio

Tintura de almizcle

R. "Lavativa".

Una vez terminado el parto se llevaba a cabo un lavado vaginal con una solución antiséptica (agua fenicada al 3 por 100, o bicloruro de mercurio al 1 por 1.000), el cual debía repetirse dos o tres veces diarias.

En las primeras horas del puerperio se ofrecía a la mujer agua de azúcar, agua con leche o con viro, infusiones ligeras de tilo o camomilla, a condición de que estuvieran a una temperatura conveniente. Al octavo día la pacietne podía abandonar el lecho y colocarse en una poltrona u otro asiento cómodo, durante una - dos horas. A los quince días del parto se le permitía salir hasta la pieza vecina.

Ya para esta época el Hospital San Juan de Dios contaba con una sala o servicio de maternidad, a cargo de un practicante designado por la Facultad de Medicina, según reza el artículo 25, capítulo VI, del Reglamento vigente en 1891: "Para el servicio científico del Hospital San Juan de Dios habrá ocho practicantes, distribuídos así: dos para cada una de las Clínicas de Patología General, de Patología Interna y de Patología Externa, uno para la Clínica Obstetrical e Infantil y uno para el servicio de sifilíticas". Pese a que entre la lista de asignaturas de la Facultad figuran aparte la Ginecología y la Obstetricia, en la práctica la primera de ellas se incluía dentro de la Patología Externa, pero para efecto de matrícula y exámenes formaba un solo curso con la Clínica Obstétrica e Infantil.

Sin ánimo de criticar los procederes de aquellos años sino con la intención de rendir un homenaje a los 
primeros practicantes del servicio de maternidad, vamos a transcribir fielmente el relato de uno de los casos que sirvieron para elaborar su tesis de grado Campo Elías Corredor, en 1892.

"El 17 de Agosto de 1892 -diceentra al servicio de maternidad Catalina Olaya, mujer de buena estatu$\mathrm{ra}$, de constitución vigorosa y de temperamento sanguíneo. Los deudos la traen sumergida en un coma profundo y refieren que desde hace un mes tiene edemas en los miembros inferiores, que en la noche anterior tuvo cinco o seis accesos convulsivos y por último que se halla en el noveno mes de embarazo. Mientras la examino estalla un ataque de eclampsia sumamente fuerte, calma el período convulsivo, pero queda cianosada y sin respiración. Tiene un edema general, el vientre es muy voluminoso, llega al reborde de las costillas; la cabeza fetal se halla en la fosa ilíaca izquierda, las pequeñas partes al lado derecho, los ruidos del corazón del feto se perciben por auscultación al lado izquierdo, debajo del ombligo. El tacto muestra un cuello alto, que apenas permite la introducción de la última falange del dedo índice. Se diagnostica un embarazo, una presentación cefálica, una eclampsia y un feto vivo. Se procede a tratarla. Mi amigo Nicolás Buendía extrae 500 gramos de sangre de la basílica izquierda. Los accesos ceden por algún tiempo, pero a las seis de la tarde estallan de nuevo y con una intensidad muy grande. La cloroformizo hasta las diez de la noche, ceden los ataques; examinado el cuello no presenta ninguna dilatación. A las once de la noche, no obstante el cloroformo, se repiten las convulsiones, son muy intensas y la asfixia que sigue a los accesos, es muy marcada. El cuello empieza a cilatarse. Mi amigo
Manuel Mejía me acompaña y resolvemos operarla.

Fuí en busca del Profesor Buendía, y no estaba en casa. Cuando volví la hallé sumergida en un coma profundo, el cuello está apenas dilatado, pero felizmente es bastante dilatable. La posición de la cabeza es la primera de Naegel, Mejía se encarga del cloroformo y yo del fórceps. La cabeza fetal está alta y acompañada por una mano. La aplicación del instrumento es larga y difícil. Los ruidos redoblados del corazón del niño han desaparecido. Después de varias tracciones, logramos extraerlo. Se le dan todos los cuidados pero no vuelve a la vida".

Pero conozcamos cuál era el aspecto y cómo funcionaba la Maternidad donde actuaban estos heroicos practicantes. Por la descripción que de ella hiciera el doctor Julio Manrique, sabemos que las mujeres que entraban allí tenían todas su parto en una misma cama de trabajo, cuyas ropas consistían en un jergón de paja recubierto con una piel curtida o vaqueta, "piel en la cual han parido - son sus palabras - ya muchas generaciones y apenas es enjugada después de cada parto con una esponja que nunca ha sido ni siquiera hervida". Concluído el alumbramiento se hacía el consabido lavado vaginal con solución antiséptica, utilizando siempre la misma cánula, y se dejaba a la mujer por espacio de una hora en reposo y observación; luego se trasladaba a la sala común.

Esta "sala común" tenía quince camas o cujas, separadas entre sí por un espacio de un metro, en el cual se improvisaban otras cuando aquellas estaban ocupadas. Con mucha frecuencia se hacinaban allí veinticuatro y más enfermas. "Al entrar a esta sala en las horas de la noche - sigue 
contando el doctor Manrique- se percibe una fetidez sui generis, inolvidable mezcla de loquios en descomposición y excreciones cutáneas en personas desaseadas".

La mortalidad materna era del 5 por 100 y el 62 por 100 de las puérperas presentaban reacción febril. Hay noticias de que en Enero de 1899 se presentó una violenta epidemia de fiebre puerperal que mató tantas madres cuantas había en la maternidad. El profesor Buendía canceló entonces el servicio, de manera temporal. Razón tenía el doctor Julio Manrique para protestar con estas palabras: "La fundación de un pabellón de Maternidad es una necesidad social de primer orden. Hay que cerrar aquel foco de infección, que es una verdadera amenaza para todas las mujeres que dan a luz en la ciudad, y que por ahora no llena ninguna de las condiciones requeridas para un servicio de esta naturaleza".

Pese a que, como anotamos atrás, la medicina y los médicos iban conquistando lentamente para sí el arte obstétrico, este seguía siendo patrimonio de ignaras comadres. "Fuera de unas pocas aficionadas -escribió Enrique Sánchez- que sin previos estudios teóricos han asistido durante un tiempo insuficiente a las conferencias prácticas dictadas en la maternidad por los eminentes profesores doctores Leoncio Barreto y José María Buendía, y en donde han aprendido lo más esencial de las atribuciones de las parteras, las demás son mujeres ignorantes llenas de achaques, como ellas mismas dicen, más dignas de recibir una pensión de jubilación que los honorarios correspondientes al ejercicio de una profesión que no pueden desempeñar, pues una de las cosas que en ella se requiere para su estricto cumplimiento, es muy buena salud. Toda su ciencia consiste en hartar a la parturiente de infusiones y decocciones de hierbas y otras sustancias sin acción terapéutica conocida o inerte, cuando no contraria al fin que se debe tratar de alcanzar...

En los casos distócicos utilizan medidas tales como tomar vivos y abrir por la cara anterior del cuerpo, de extremo a extremo, pichones, ratones, gatos $u$ otros animales de la laya $y$ colocarlos, palpitantes aún, en el hueco epigástrico, en la nuca o en las pantorrillas de la enferma, con la seguridad de un éxito completo".

Nos entra sospecha, después de conocer los recursos terapéuticos que empleaban esas achacosas mujeres, que muy pocas leyeron la obrita dedicada a ellas y que, a petición de la Sociedad Filantrópica, escribiera y publicara en Bogotá el doctor Gabriel D. Ujueta en 1881; en cambio, muchas debieron formarse en la escuela de Telmo A. Romero a través de su obra magna "El Bien General", editada en Caracas en 1885 pero muy difundida en Colombia. Este don Telmo Romero fue un curandero que anduvo entre las tribus indígenas que habitaban la parte oriental colombiana y la occidental de Venezuela y que, como diría el Virrey Ezpeleta, a vuelta de un cierto casual que publicó y lo acreditó, tuvo a su favor la confianza de muchas gentes. Oigamos algunas de sus enseñanzas en cuestión de obstetricia:

"Fara saber el estado interesante o normal de las señoras", se les dan dos onzas de miel de abejas mezcladas con una de acíbar de zábila, tomando inmediatamnete un vaso de agua natural de estanque. Si no aparece el período, se hará que orinen en una vasija aseada y dentro de ella se colocará un dedal de plata o cle 
acero y si al cabo de tres horas no se ha enmohecido, es señal evidente de que no se encuentre encinta.

"Para contener los abortos" se prepara una orchata compuesta de cuatro onzas de semillas de ajonjolí tostado y pulverizado, cuatro de la segunda corteza de yagrumo, una y media libra de azúcar y ocho onzas de miel de abejas en la cantidad de agua suficiente para que pueda quedar de regular grosor; luego que todo haya hervido lo suficiente se deja reposar para trasegársela en botellas y de ello tomarse un vaso diario, poco después del medio día, agregándole a cada toma una dracma de cuajo, el cual debe ser escogido particularmente de toro negro. Esta bebida debe tomarse por espacio de veinte días.

"Para facilitar los partos difíciles" es muy provechoso tomar dos nudos tostados y pulverizados del espinazo del pez eléctrico conocido con el nombre de temblador. También puede usarse una bebida compuesta de seis onzas de aceite de olivas, una de azafrán pulverizado, el zumo que produzcan cuatro onzas de estiércol fresblanco, de la cual se dará una onza cada cuarto de hora hasta que se haya desembarazado.

"Para los entuertos", luego de desecar una molleja de gallina, el pellejo que se encuentra dentro se pulveriza y se disuelve en cuatro onzas de vino seco, todo lo cual se administra en forma de bebida.

Vemos, pues, por las anteriores noticias, en manos de quiénes se encontraba el arte obstétrico en Bogotá al finalizar el Siglo XIX: de unos pocos pero muy brillantes exponentes de la Medicina Colombiana, y de una legión de parteras, mezcla de madre chibcha y comadre española. La ginecología, en tanto, reducida a la exéresis, era del dominio de operadores audaces, quienes a la par que extir- paban úteros y ovarios, comenzaban a forjar la historia de nuestra cirugía.

\section{BIBLIOGRAFIA}

1 BONILLA-NAAR, ALFONSO: "Precursores de la cirugía en Colombia". Ed. Antares. Bogotá, 1954

2 CORDOVEZ, JOSE M. "Reminiscencias de Santafé de Bogotá". Ed. Aguilar. Madrid, 1957.

3 CORPAS, JUAN N.: "Resumen histórico de la enseñanza de la Medicina y de las distintas escuelas que han existido en Bogotá desde la época de la Colonia hasta la fundación de la actual Facultad de Medicina y Ciencias Naturales de la Universidad Nacional". Rev. de la Facultad de Medicina (U. N.) Vol. I, No 1, 1932.

4 CORREDOR, CAMPO E. "Contribució para el estudio de los partos difíciles. Eclampsia puerperal". Tesis de grado. Imprenta de Antonio M. Silvestre. Bogotá, 1892.

5 IBAÑEZ, PEDRO M. "Memorias de la historia de la Medicina en Santafé". Imprenta de vapor de Zalamea Hnos. Bogotá, 1884.

6 La Lanceta. No 1, Abril de 1852.

7 MAHECHA Z., FROILAN. "Distocia disodínica". Tesis de grado. Facultad de Medicina (U.N.). Bogotá, 1896.

8 MANRIQUE, JULIO. "Infecciones puerperales en la Naternidad de Bogotá". Tesis de grado. Imprenta Nacional. Bogotá, 1897.

9 NONTAÑA, ELISEO. "Contribución al estudio de la ovariotomía en Colombia". Imprenta de Echeverría Hnos. Bogotá, 1891.

10 MUÑOZ, LAURENTINO. "Historia del Hospital de San José, 1902-1956". Imprenta del Banco de la República. Bogotá, 1958.

11 RAMIREZ L., CARLOS J. "Higiene puerperal". Tesis de grado. Imprenta La Luz. Bogotá, 1893.

12 Reglamento para la Facultad de Nedicina (Universidad Nacional). Casa Ed. de Rivas y Cía. Bogotá, 1891.

13 RONERO, TELMO A.: "El Bien General". Imprenta Nacional. Caracas, 1885.

14 SANCHEZ C., ENRIQUE: "Necesidad de reglamentar en Colombia el ejercicio de la profesión obstetrical". Tesis de grado. Imprenta La Nación. 1892.

15 SANNIGUED, JOSE P. "Al Congreso de los Estados Unidos de Colombia en sus sesiones ordinarias de 1869". Imprenta de Gaitán. Bogotá. 1869.

16 SERRANO, JOSE J. “Higienización de Bogotá". Tesis de grado. Imprenta de Vapor de Zalamea Hnos. Bogotá, 1899.

17 SORIANO LLERAS, ANDRES. "Crónica del Hospital de San Juan de Dios, 1564-1869". Italgraf, Ltda. Bogotá, 1964. 